\title{
Norm inequalities for operators related to the Cauchy-Schwarz and Heinz inequalities
}

Jianguo Zhao ${ }^{1 *}$, Hongmei Xie ${ }^{1}$, Junliang $\mathrm{Wu}^{2}$ and Keshe $\mathrm{Ni}^{1}$

\section{*Correspondence:}

jgzhao_dj@163.com

${ }^{1}$ College of Science, Shihezi

University, Shihezi, 832003, P.R.

China

Full list of author information is

available at the end of the article

\begin{abstract}
We present some refinements of the Cauchy-Schwarz and Heinz inequalities for operators by utilizing a refinement of the Hermite-Hadamard inequality.
\end{abstract}

MSC: $15 \mathrm{~A} 45 ; 15 \mathrm{~A} 60$

Keywords: Hermite-Hadamard inequality; norm inequality; Cauchy-Schwarz inequality; Heinz inequality

\section{Introduction}

Let $B(\mathcal{H})$ be the $C^{*}$-algebra of all bounded linear operators on a complex separable Hilbert space $\mathcal{H}$. Let $\|\cdot\|$ denote a unitarily invariant norm defined on a two-sided ideal $\tau_{\|\cdot\|}$ that is included in $C_{\infty}$ (the set of compact operators); it has the basic property $\|U A V\|=\|A\|$ for every $A \in \tau_{\|\cdot\|}$ and all unitary operators $U, V \in B(\mathcal{H})$. For $A \in B(\mathcal{H}),|A|=\left(A^{*} A\right)^{\frac{1}{2}}$, where $A^{*}$ is the conjugate operator of $A$.

Hiai and Zhan [1] proved that if $A, B, X \in B(\mathcal{H})$ with $A, B$ positive and $X \in \tau_{\|\cdot\|}, r>0$, then the function $f(v)=\left\|\left|A^{v} X B^{1-v}\right|^{r}\right\| \cdot\left\|\left|A^{1-v} X B^{v}\right|^{r}\right\|$ is convex on the interval $[0,1]$ and attains its minimum at $v=\frac{1}{2}$ maximum at $v=0$ and $v=1$. Consequently, it is decreasing on $\left[0, \frac{1}{2}\right]$ and increasing on $\left[\frac{1}{2}, 1\right]$; moreover, $f(v)=f(1-v)$ for $v \in[0,1]$. Thus for every unitarily invariant norm $\|\cdot\|$, we have the operator inequality

$$
\left\|\left|A^{\frac{1}{2}} X B^{\frac{1}{2}}\right|^{r}\right\|^{2} \leq\left\|\left|A^{v} X B^{1-v}\right|^{r}\right\| \cdot\left\|\left|A^{1-v} X B^{v}\right|^{r}\right\| \leq\left\||A X|^{r}\right\| \cdot\left\||X B|^{r}\right\|
$$

for $A, B, X \in B(\mathcal{H})$ with $A, B$ positive, $X \in \tau_{\|\cdot\|}, r>0$, and $v \in[0,1]$. The inequality $\left\|\left|A^{\frac{1}{2}} X B^{\frac{1}{2}}\right|^{r}\right\|^{2} \leq\left\||A X|^{r}\right\| \cdot\left\||X B|^{r}\right\|$ is often called the Cauchy-Schwarz norm inequality due to Bhatia and Davis [2].

The Heinz means for operators are defined by $g(v)=\left\|A^{v} X B^{1-v}+A^{1-v} X B^{v}\right\|$ for $A, B, X \in$ $B(\mathcal{H})$ with $A, B$ positive, $X \in \tau_{\|\cdot\| \|}$, and $v \in[0,1]$, where $\|\cdot\|$ is a unitarily invariant norm. Bhatia and Davis [3] obtained the inequality

$$
2\left\|A^{\frac{1}{2}} X B^{\frac{1}{2}}\right\| \leq\left\|A^{v} X B^{1-v}+A^{1-v} X B^{v}\right\| \leq\|A X+X B\|
$$

for $A, B, X \in B(\mathcal{H})$ with $A, B$ positive, $X \in \tau_{\|\cdot\|}$, and $v \in[0,1]$. In fact, they proved that $g(v)=$ $\left\|A^{v} X B^{1-v}+A^{1-v} X B^{v}\right\|$ is a convex function of $v$ on the interval [0,1], attains its minimum at $v=\frac{1}{2}$ and maximum at $v=0$ and $v=1$. Hence, it is decreasing on $\left[0, \frac{1}{2}\right]$ and increasing

(c) Zhao et al. 2016. This article is distributed under the terms of the Creative Commons Attribution 4.0 International License (http://creativecommons.org/licenses/by/4.0/), which permits unrestricted use, distribution, and reproduction in any medium, provided you give appropriate credit to the original author(s) and the source, provide a link to the Creative Commons license, and indicate if changes were made. 
on $\left[\frac{1}{2}, 1\right]$; moreover, $g(v)=g(1-v)$ for $v \in[0,1]$. The second inequality in $(2)$ is one of the most essential inequalities in operator theory, which is often called the Heinz inequality.

Recently, using the convexity of the function $f(v)=\left\|\left|A^{v} X B^{1-v}\right|^{r}\right\| \cdot\left\|\left|A^{1-v} X B^{v}\right|^{r}\right\|(v \in$ $[0,1])$, Burqan [4], Theorem 2, obtained a refinement of inequality (1): Let $A, B, X \in B(\mathcal{H})$ with $A, B$ positive and $X \in \tau_{\|\cdot\|}$. Then, for every unitarily invariant norm $\|\cdot\|$,

$$
\begin{aligned}
\left\|\left|A^{\frac{1}{2}} X B^{\frac{1}{2}}\right|^{r}\right\|^{2} & \leq\left\|\left|A^{\frac{2 v+1}{4}} X B^{\frac{3-2 v}{4}}\right|^{r}\right\| \cdot\left\|\left|A^{\frac{3-2 v}{4}} X B^{\frac{2 v+1}{4}}\right|^{r}\right\| \\
& \leq \frac{1}{|1-2 v|}\left|\int_{v}^{1-v}\left\|\left|A^{\mu} X B^{1-\mu}\right|^{r}\right\| \cdot\left\|\left|A^{1-\mu} X B^{\mu}\right|^{r}\right\| d \mu\right| \\
& \leq \frac{1}{2}\left[\left\|\left|A^{v} X B^{1-v}\right|^{r}\right\| \cdot\left\|\left|A^{1-v} X B^{v}\right|^{r}\right\|+\left\|\left|A^{\frac{1}{2}} X B^{\frac{1}{2}}\right|^{r}\right\|^{2}\right] \\
& \leq\left\|\left|A^{v} X B^{1-v}\right|^{r}\right\| \cdot\left\|\left|A^{1-v} X B^{v}\right|^{r}\right\| \\
& \leq\left\||A X|^{r}\right\| \cdot\left\||X B|^{r}\right\|,
\end{aligned}
$$

where $v \in[0,1]$ and $r>0$.

Using the convexity of the function $g(v)=\left\|A^{v} X B^{1-v}+A^{1-v} X B^{v}\right\|(v \in[0,1])$, Feng [5], Theorem 1 , obtained a refinement of the first inequality in (2): Let $A, B, X \in B(\mathcal{H})$ with $A, B$ positive and $X \in \tau_{\|\cdot\|}$. Then, for every unitarily invariant norm $\|\cdot\|$,

$$
\begin{aligned}
2\left\|A^{\frac{1}{2}} X B^{\frac{1}{2}}\right\| & \leq \frac{1}{|1-2 v|}\left|\int_{v}^{1-v}\left\|A^{\mu} X B^{1-\mu}+A^{1-\mu} X B^{\mu}\right\| d \mu\right| \\
& \leq \frac{1}{2}\left[\left\|A^{v} X B^{1-v}+A^{1-v} X B^{v}\right\|+2\left\|A^{\frac{1}{2}} X B^{\frac{1}{2}}\right\|\right] \\
& \leq\left\|A^{v} X B^{1-v}+A^{1-v} X B^{v}\right\|,
\end{aligned}
$$

where $v \in[0,1]$.

In this note, we present some refinements of norm inequalities (1), (2), (3), and (4), also using the convexity of the functions $f$ and $g$.

\section{Main results}

In this section, we mainly present some refinements of the Cauchy-Schwarz and Heinz inequalities for operators. To achieve our goal, we need some preparations.

Let $f: I \rightarrow R$ be a real-valued convex function on the interval $I \subseteq R$. Let $a, b \in I$ with $a<b$. The inequality

$$
f\left(\frac{a+b}{2}\right) \leq \frac{1}{b-a} \int_{a}^{b} f(x) d x \leq \frac{f(a)+f(b)}{2}
$$

is well known as the Hermite-Hadamard inequality, which includes a basic property of convex functions.

Now, we present some lemmas.

Lemma 2.1 Let $f$ be a real-valued convex function on the interval $[a, b]$. Then

$$
\frac{1}{4}\left(f\left(\frac{7 a+b}{8}\right)+f\left(\frac{5 a+3 b}{8}\right)+f\left(\frac{3 a+5 b}{8}\right)+f\left(\frac{a+7 b}{8}\right)\right) \leq \frac{1}{b-a} \int_{a}^{b} f(x) d x
$$


Proof Thanks to the Hermite-Hadamard's inequality (5), we obtain

$$
f\left(\frac{7 a+b}{8}\right)=f\left(\frac{a+\frac{3 a+b}{4}}{2}\right) \leq \frac{4}{b-a} \int_{a}^{\frac{3 a+b}{4}} f(x) d x
$$

and

$$
f\left(\frac{5 a+3 b}{8}\right)=f\left(\frac{\frac{3 a+b}{4}+\frac{a+b}{2}}{2}\right) \leq \frac{4}{b-a} \int_{\frac{3 a+b}{4}}^{\frac{a+b}{2}} f(x) d x
$$

Inequalities (7) and (8) give

$$
f\left(\frac{7 a+b}{8}\right)+f\left(\frac{5 a+3 b}{8}\right) \leq \frac{4}{b-a} \int_{a}^{\frac{a+b}{2}} f(x) d x
$$

Similarly, due to the convexity of $f$ on the interval $\left[\frac{a+b}{2}, b\right]$, we have

$$
f\left(\frac{3 a+5 b}{8}\right)+f\left(\frac{a+7 b}{8}\right) \leq \frac{4}{b-a} \int_{\frac{a+b}{2}}^{b} f(x) d x
$$

Hence, by inequalities (9) and (10) we deduce

$$
\begin{aligned}
& \frac{1}{4}\left(f\left(\frac{7 a+b}{8}\right)+f\left(\frac{5 a+3 b}{8}\right)+f\left(\frac{3 a+5 b}{8}\right)+f\left(\frac{a+7 b}{8}\right)\right) \\
& \quad \leq \frac{1}{b-a} \int_{a}^{b} f(x) d x
\end{aligned}
$$

This completes the proof.

Remark 2.2 Let $f$ be a real-valued convex function on the interval $[a, b]$. By the convexity of $f$ on the interval $\left[a, \frac{a+b}{2}\right]$ we get

$$
\begin{aligned}
f\left(\frac{3 a+b}{4}\right) & =f\left(\frac{1}{2}\left(\frac{7 a+b}{8}\right)+\frac{1}{2}\left(\frac{5 a+3 b}{8}\right)\right) \\
& \leq \frac{1}{2} f\left(\frac{7 a+b}{8}\right)+\frac{1}{2} f\left(\frac{5 a+3 b}{8}\right) .
\end{aligned}
$$

Similarly, due to the convexity of $f$ on the interval $\left[\frac{a+b}{2}, b\right]$, we have

$$
f\left(\frac{a+3 b}{4}\right) \leq \frac{1}{2} f\left(\frac{3 a+5 b}{8}\right)+\frac{1}{2} f\left(\frac{a+7 b}{8}\right)
$$

Hence, inequality (6) is a refinement of the inequality

$$
\frac{1}{2}\left(f\left(\frac{3 a+b}{4}\right)+f\left(\frac{a+3 b}{4}\right)\right) \leq \frac{1}{b-a} \int_{a}^{b} f(x) d x
$$

obtained by Burqan [4], Lemma 1. 
Lemma 2.3 Let $f$ be a real-valued convex function on the interval $[a, b]$. Then

$$
\begin{aligned}
\frac{1}{b-a} \int_{a}^{b} f(x) d x \leq & \frac{1}{8}\left(f(a)+2 f\left(\frac{3 a+b}{4}\right)+2 f\left(\frac{a+b}{2}\right)\right. \\
& \left.+2 f\left(\frac{a+3 b}{4}\right)+f(b)\right) .
\end{aligned}
$$

Proof By the Hermite-Hadamard's inequality (5) we obtain

$$
\begin{aligned}
\frac{1}{b-a} \int_{a}^{b} f(x) d x= & \frac{1}{b-a}\left\{\int_{a}^{\frac{3 a+b}{4}} f(x) d x+\int_{\frac{3 a+b}{4}}^{\frac{a+b}{2}} f(x) d x\right. \\
& \left.+\int_{\frac{a+b}{2}}^{\frac{a+3 b}{4}} f(x) d x+\int_{\frac{a+3 b}{4}}^{b} f(x) d x\right\} \\
\leq & \frac{1}{b-a}\left\{\frac{f(a)+f\left(\frac{3 a+b}{4}\right)}{2} \cdot \frac{b-a}{4}+\frac{f\left(\frac{3 a+b}{4}\right)+f\left(\frac{a+b}{2}\right)}{2} \cdot \frac{b-a}{4}\right. \\
& \left.+\frac{f\left(\frac{a+b}{2}\right)+f\left(\frac{a+3 b}{4}\right)}{2} \cdot \frac{b-a}{4}+\frac{f\left(\frac{a+3 b}{4}\right)+f(b)}{2} \cdot \frac{b-a}{4}\right\} \\
= & \frac{1}{8}\left(f(a)+2 f\left(\frac{3 a+b}{4}\right)+2 f\left(\frac{a+b}{2}\right)+2 f\left(\frac{a+3 b}{4}\right)+f(b)\right) .
\end{aligned}
$$

This completes the proof.

Remark 2.4 Let $f$ be a real-valued convex function on the interval $[a, b]$. Thanks to the convexity of $f$ on the interval $\left[a, \frac{a+b}{2}\right]$ and $\left[\frac{a+b}{2}, b\right]$, respectively, we obtain

$$
f\left(\frac{3 a+b}{4}\right)=f\left(\frac{a+\frac{a+b}{2}}{2}\right) \leq \frac{f(a)+f\left(\frac{a+b}{2}\right)}{2}
$$

and

$$
f\left(\frac{a+3 b}{4}\right)=f\left(\frac{\frac{a+b}{2}+b}{2}\right) \leq \frac{f(b)+f\left(\frac{a+b}{2}\right)}{2} .
$$

Hence, combining inequalities (14) and (15), we get

$$
\begin{aligned}
& \frac{1}{2}\left(f(a)+2 f\left(\frac{3 a+b}{4}\right)+2 f\left(\frac{a+b}{2}\right)+2 f\left(\frac{a+3 b}{4}\right)+f(b)\right) \\
& \quad \leq\left(f(a)+2 f\left(\frac{a+b}{2}\right)+f(b)\right) .
\end{aligned}
$$

Hence, inequality (13) is a refinement of the inequality

$$
\frac{1}{b-a} \int_{a}^{b} f(x) d x \leq \frac{1}{4}\left(f(a)+2 f\left(\frac{a+b}{2}\right)+f(b)\right)
$$

obtained by Feng [5], Lemma 2.

Combining Lemmas 2.1 and 2.3, we obtain the following lemma. 
Lemma 2.5 Let $f$ be a real-valued convex function on the interval $[a, b]$. Then

$$
\begin{aligned}
\frac{1}{4}\left(f\left(\frac{7 a+b}{8}\right)+f\left(\frac{5 a+3 b}{8}\right)+f\left(\frac{3 a+5 b}{8}\right)+f\left(\frac{a+7 b}{8}\right)\right) \\
\leq \frac{1}{b-a} \int_{a}^{b} f(x) d x \\
\leq \frac{1}{8}\left(f(a)+2 f\left(\frac{3 a+b}{4}\right)+2 f\left(\frac{a+b}{2}\right)\right. \\
\left.\quad+2 f\left(\frac{a+3 b}{4}\right)+f(b)\right) .
\end{aligned}
$$

Remark 2.6 By inequalities (11), (12), and (16) it is easy to see that inequality (17) is a refinement of inequality (5).

Based on Lemma 2.5, we obtain the following theorems.

Theorem 2.7 Let $\|\cdot\|$ be a unitarily invariant norm, and $A, B, X \in B(\mathcal{H})$ with $A, B$ positive and $X \in \tau_{\|\cdot\| \cdot}$. Then

$$
\begin{aligned}
& \frac{1}{2}\left\{\left\|\left|A^{\frac{6 v+1}{8}} X B^{\frac{7-6 v}{8}}\right|^{r}\right\| \cdot\left\|\left|A^{\frac{7-6 v}{8}} X B^{\frac{6 v+1}{8}}\right|^{r}\right\|+\left\|\left|A^{\frac{2 v+3}{8}} X B^{\frac{5-2 v}{8}}\right|^{r}\right\| \cdot\left\|\left|A^{\frac{5-2 v}{8}} X B^{\frac{2 v+3}{8}}\right|^{r}\right\|\right\} \\
& \leq \frac{1}{|1-2 v|}\left|\int_{v}^{1-v}\left\|\left|A^{x} X B^{1-x}\right|^{r}\right\| \cdot\left\|\left|A^{1-x} X B^{x}\right|^{r}\right\| d x\right| \\
& \leq \frac{1}{4}\left\{\left\|\left|A^{v} X B^{1-v}\right|^{r}\right\| \cdot\left\|\left|A^{1-v} X B^{v}\right|^{r}\right\|+\left\|\left|A^{\frac{1}{2}} X B^{\frac{1}{2}}\right|^{r}\right\|^{2}\right. \\
&\left.+2\left\|\left|A^{\frac{2 v+1}{4}} X B^{\frac{3-2 v}{4}}\right|^{r}\right\| \cdot\left\|\left|A^{\frac{3-2 v}{4}} X B^{\frac{2 v+1}{4}}\right|^{r}\right\|\right\}
\end{aligned}
$$

where $v \in[0,1] \backslash\left\{\frac{1}{2}\right\}$ and $r>0$.

Proof We first consider the case $0 \leq v<\frac{1}{2}$. Applying Lemma 2.5 to the function $f(x)=$ $\left\|\left|A^{x} X B^{1-x}\right|^{r}\right\| \cdot\left\|\left|A^{1-x} X B^{x}\right|^{r}\right\|$ on the interval $[v, 1-v]$ and $f(x)=f(1-x)$ for $x \in[0,1]$, we obtain

$$
\begin{aligned}
\frac{1}{2}\left\{f\left(\frac{6 v+1}{8}\right)+f\left(\frac{2 v+3}{8}\right)\right\} & \leq \frac{1}{1-2 v} \int_{v}^{1-v} f(x) d x \\
& \leq \frac{1}{4}\left\{f(v)+2 f\left(\frac{2 v+1}{4}\right)+f\left(\frac{1}{2}\right)\right\} .
\end{aligned}
$$

Thus, we get inequality (18) for $0 \leq v<\frac{1}{2}$.

Similarly, when $\frac{1}{2}<v \leq 1$, applying Lemma 2.5 to the function $f$ on the interval $[1-v, v]$ and $f(x)=f(1-x)$ for $x \in[0,1]$, we obtain

$$
\begin{aligned}
\frac{1}{2}\left\{f\left(\frac{6 v+1}{8}\right)+f\left(\frac{2 v+3}{8}\right)\right\} & \leq \frac{1}{2 v-1} \int_{1-v}^{v} f(x) d x \\
& \leq \frac{1}{4}\left\{f(v)+2 f\left(\frac{2 v+1}{4}\right)+f\left(\frac{1}{2}\right)\right\} .
\end{aligned}
$$

Hence, we get inequality (18) for $\frac{1}{2}<v \leq 1$. 
This completes the proof.

The next theorem is a refinement of Heinz's norm inequalities.

Theorem 2.8 Let $\|\cdot\|$ be a unitarily invariant norm, and $A, B, X \in B(\mathcal{H})$ with $A, B$ positive and $X \in \tau_{\|\cdot\| \cdot}$. Then

$$
\begin{aligned}
& \frac{1}{2}\left\{\left\|A^{\frac{6 v+1}{8}} X B^{\frac{7-6 v}{8}}+A^{\frac{7-6 v}{8}} X B^{\frac{6 v+1}{8}}\right\|+\left\|A^{\frac{2 v+3}{8}} X B^{\frac{5-2 v}{8}}+A^{\frac{5-2 v}{8}} X B^{\frac{2 v+3}{8}}\right\|\right\} \\
& \leq \frac{1}{|1-2 v|}\left|\int_{v}^{1-v}\left\|A^{x} X B^{1-x}+A^{1-x} X B^{x}\right\| d x\right| \\
& \leq \frac{1}{4}\left\{\left\|A^{v} X B^{1-v}+A^{1-v} X B^{v}\right\|+2\left\|A^{\frac{1}{2}} X B^{\frac{1}{2}}\right\|\right. \\
&\left.+2\left\|A^{\frac{2 v+1}{4}} X B^{\frac{3-2 v}{4}}+A^{\frac{3-2 v}{4}} X B^{\frac{2 v+1}{4}}\right\|\right\},
\end{aligned}
$$

where $v \in[0,1] \backslash\left\{\frac{1}{2}\right\}$.

Proof Replacing $f(x)=\left\|\left|A^{x} X B^{1-x}\right|^{r}\right\| \cdot\left\|\left|A^{1-x} X B^{x}\right|^{r}\right\|$ by $g(x)=\left\|A^{x} X B^{1-x}+A^{1-x} X B^{x}\right\|$ in the proof of Theorem 2.7, we get the desired result.

Remark 2.9 Putting $f(x)=\left\|\left|A^{x} X B^{1-x}\right|^{r}\right\| \cdot\left\|\left|A^{1-x} X B^{x}\right|^{r}\right\|(x \in[0,1])$, where $\|\cdot\|$ is a unitarily invariant norm, $A, B, X \in B(\mathcal{H})$ with $A, B$ positive, $X \in \tau_{\|\cdot\|}$, and $r>0$. By the convexity of $f$ we have $f\left(\frac{2 v+1}{4}\right) \leq \frac{1}{2}\left\{f\left(\frac{6 v+1}{8}\right)+f\left(\frac{2 v+3}{8}\right)\right\}$ and $\frac{1}{4}\left\{f(v)+2 f\left(\frac{2 v+1}{4}\right)+f\left(\frac{1}{2}\right)\right\} \leq \frac{1}{2}\left\{f(v)+f\left(\frac{1}{2}\right)\right\}$ for $v \in[0,1] \backslash\left\{\frac{1}{2}\right\}$. So, norm inequality (18) is a refinement of inequality (3) obtained by Burqan [4], Theorem 2.

Remark 2.10 Putting $g(x)=\left\|A^{x} X B^{1-x}+A^{1-x} X B^{x}\right\|(x \in[0,1])$, where $\|\cdot\|$ is a unitarily invariant norm, and $A, B, X \in B(\mathcal{H})$ with $A, B$ positive and $X \in \tau_{\|\cdot\|}$. By the convexity of $g$ we have $g\left(\frac{2 v+1}{4}\right) \leq \frac{1}{2}\left\{g\left(\frac{6 v+1}{8}\right)+g\left(\frac{2 v+3}{8}\right)\right\}$ and $\frac{1}{4}\left\{g(v)+2 g\left(\frac{2 v+1}{4}\right)+g\left(\frac{1}{2}\right)\right\} \leq \frac{1}{2}\left\{g(v)+g\left(\frac{1}{2}\right)\right\}$ for $v \in[0,1] \backslash\left\{\frac{1}{2}\right\}$. Hence, norm inequality (19) is a refinement of inequality (4) obtained by Feng [5], Theorem 1.

For $0<v<1$, applying Lemma 2.5 to the convex functions $f(x)=\left\|\left|A^{x} X B^{1-x}\right|^{r}\right\|$. $\left\|\left|A^{1-x} X B^{x}\right|^{r}\right\|$ and $g(x)=\left\|A^{x} X B^{1-x}+A^{1-x} X B^{x}\right\|$ on the interval $[0, v]$ when $0<v \leq \frac{1}{2}$ and on the interval $[v, 1]$ when $\frac{1}{2} \leq v<1$, respectively, we obtain the following theorems, where $\|\cdot\|$ is a unitarily invariant norm, $A, B, X \in B(\mathcal{H})$ with $A, B$ positive and $X \in \tau_{\|\cdot\|}$, and $r>0$.

Theorem 2.11 Let $\|\cdot\|$ be a unitarily invariant norm, and $A, B, X \in B(\mathcal{H})$ with $A, B$ positive and $X \in \tau_{\|\cdot\| \cdot}$. Then

(i) for $0<v \leq \frac{1}{2}$,

$$
\begin{aligned}
\frac{1}{4}\left\{\left\|\left|A^{\frac{v}{8}} X B^{\frac{8-v}{8}}\right|^{r}\right\| \cdot\left\|\left|A^{\frac{8-v}{8}} X B^{\frac{v}{8}}\right|^{r}\right\|+\left\|\left|A^{\frac{3 v}{8}} X B^{\frac{8-3 v}{8}}\right|^{r}\right\|\right. \\
\cdot\left\|\left|A^{\frac{8-3 v}{8}} X B^{\frac{3 v}{8}}\right|^{r}\right\|+\left\|\left|A^{\frac{5 v}{8}} X B^{\frac{8-5 v}{8}}\right|^{r}\right\| \cdot\left\|\left|A^{\frac{8-5 v}{8}} X B^{\frac{5 v}{8}}\right|^{r}\right\| \\
\left.+\left\|\left|A^{\frac{7 v}{8}} X B^{\frac{8-7 v}{8}}\right|^{r}\right\| \cdot\left\|\left|A^{\frac{8-7 v}{8}} X B^{\frac{7 v}{8}}\right|^{r}\right\|\right\} \\
\leq \frac{1}{v} \int_{0}^{v}\left\|\left|A^{x} X B^{1-x}\right|^{r}\right\| \cdot\left\|\left|A^{1-x} X B^{x}\right|^{r}\right\| d x
\end{aligned}
$$




$$
\begin{aligned}
\leq & \frac{1}{8}\left\{\left\||A X|^{r}\right\| \cdot\left\||X B|^{r}\right\|+2\left\|\left|A^{\frac{v}{4}} X B^{\frac{4-v}{4}}\right|^{r}\right\| \cdot\left\|\left|A^{\frac{4-v}{4}} X B^{\frac{v}{4}}\right|^{r}\right\|\right. \\
& +2\left\|\left|A^{\frac{v}{2}} X B^{\frac{2-v}{2}}\right|^{r}\right\| \cdot\left\|\left|A^{\frac{2-v}{2}} X B^{\frac{v}{2}}\right|^{r}\right\|+2\left\|\left|A^{\frac{3 v}{4}} X B^{\frac{4-3 v}{4}}\right|^{r}\right\| \\
& \left.\cdot\left\|\left|A^{\frac{4-3 v}{4}} X B^{\frac{3 v}{4}}\right|^{r}\right\|+\left\|\left|A^{v} X B^{1-v}\right|^{r}\right\| \cdot\left\|\left|A^{1-v} X B^{v}\right|^{r}\right\|\right\}
\end{aligned}
$$

(ii) for $\frac{1}{2}<v<1$,

$$
\begin{aligned}
& \frac{1}{4}\{\|\left|A^{\frac{7 v+1}{8}} X B^{\frac{7-7 v}{8}}\right|^{r}\|\cdot\|\left|A^{\frac{7-7 v}{8}} X B^{\frac{7 v+1}{8}}\right|^{r}\|+\|\left|A^{\frac{5 v+3}{8}} X B^{\frac{5-5 v}{8}}\right|^{r} \| \\
& \cdot\left\|\left|A^{\frac{5-5 v}{8}} X B^{\frac{5 v+3}{8}}\right|^{r}\right\|+\left\|\left|A^{\frac{3 v+5}{8}} X B^{\frac{3-3 v}{8}}\right|^{r}\right\| \cdot\left\|\left|A^{\frac{3-3 v}{8}} X B^{\frac{3 v+5}{8}}\right|^{r}\right\| \\
&\left.+\left\|\left|A^{\frac{v+7}{8}} X B^{\frac{1-v}{8}}\right|^{r}\right\| \cdot\left\|\left|A^{\frac{1-v}{8}} X B^{\frac{v+7}{8}}\right|^{r}\right\|\right\} \\
& \leq \frac{1}{1-v} \int_{v}^{1}\left\|\left|A^{x} X B^{1-x}\right|^{r}\right\| \cdot\left\|\left|A^{1-x} X B^{x}\right|^{r}\right\| d x \\
& \leq \frac{1}{8}\left\{\left\||A X|^{r}\right\| \cdot\left\||X B|^{r}\right\|+2\left\|\left|A^{\frac{3 v+1}{4}} X B^{\frac{3-3 v}{4}}\right|^{r}\right\| \cdot\left\|\left|A^{\frac{3-3 v}{4}} X B^{\frac{3 v+1}{4}}\right|^{r}\right\|\right. \\
&+2\left\|\left|A^{\frac{v+1}{2}} X B^{\frac{1-v}{2}}\right|^{r}\right\| \cdot\left\|\left|A^{\frac{1-v}{2}} X B^{\frac{v+1}{2}}\right|^{r}\right\|+2\left\|\left|A^{\frac{v+3}{4}} X B^{\frac{1-v}{4}}\right|^{r}\right\| \\
&\left.\cdot\left\|\left|A^{\frac{1-v}{4}} X B^{\frac{v+3}{4}}\right|^{r}\right\|+\left\|\left|A^{v} X B^{1-v}\right|^{r}\right\| \cdot\left\|\left.A^{1-v} X B^{v}\right|^{r}\right\|\right\},
\end{aligned}
$$

where $r>0$.

Theorem 2.12 Let $\|\cdot\|$ be a unitarily invariant norm, and $A, B, X \in B(\mathcal{H})$ with $A$, B positive and $X \in \tau_{\|\cdot\| \cdot}$. Then

(i) for $0<v \leq \frac{1}{2}$,

$$
\begin{aligned}
\frac{1}{4}\{\| & A^{\frac{v}{8}} X B^{\frac{8-v}{8}}+A^{\frac{8-v}{8}} X B^{\frac{v}{8}}\|+\| A^{\frac{3 v}{8}} X B^{\frac{8-3 v}{8}}+A^{\frac{8-3 v}{8}} X B^{\frac{3 v}{8}} \| \\
& \left.+\left\|A^{\frac{5 v}{8}} X B^{\frac{8-5 v}{8}}+A^{\frac{8-5 v}{8}} X B^{\frac{5 v}{8}}\right\|+\left\|A^{\frac{7 v}{8}} X B^{\frac{8-7 v}{8}}+A^{\frac{8-7 v}{8}} X B^{\frac{7 v}{8}}\right\|\right\} \\
\leq & \frac{1}{v} \int_{0}^{v}\left\|A^{x} X B^{1-x}+A^{1-x} X B^{x}\right\| d x \\
\leq & \frac{1}{8}\left\{\|A X+X B\|+2\left\|A^{\frac{v}{4}} X B^{\frac{4-v}{4}}+A^{\frac{4-v}{4}} X B^{\frac{v}{4}}\right\|\right. \\
& +2\left\|A^{\frac{v}{2}} X B^{\frac{2-v}{2}}+A^{\frac{2-v}{2}} X B^{\frac{v}{2}}\right\|+2\left\|A^{\frac{3 v}{4}} X B^{\frac{4-3 v}{4}}+A^{\frac{4-3 v}{4}} X B^{\frac{3 v}{4}}\right\| \\
& \left.+\left\|A^{v} X B^{1-v}+A^{1-v} X B^{v}\right\|\right\} ;
\end{aligned}
$$

(ii) for $\frac{1}{2}<v \leq 1$,

$$
\begin{aligned}
& \frac{1}{4}\left\{\left\|A^{\frac{7 v+1}{8}} X B^{\frac{7-7 v}{8}}+A^{\frac{7-7 v}{8}} X B^{\frac{7 v+1}{8}}\right\|+\left\|A^{\frac{5 v+3}{8}} X B^{\frac{5-5 v}{8}}+A^{\frac{5-5 v}{8}} X B^{\frac{5 v+3}{8}}\right\|\right. \\
&\left.\quad+\left\|A^{\frac{3 v+5}{8}} X B^{\frac{3-3 v}{8}}+A^{\frac{3-3 v}{8}} X B^{\frac{3 v+5}{8}}\right\|+\left\|A^{\frac{v+7}{8}} X B^{\frac{1-v}{8}}+A^{\frac{1-v}{8}} X B^{\frac{v+7}{8}}\right\|\right\} \\
& \leq \frac{1}{1-v} \int_{v}^{1}\left\|A^{x} X B^{1-x}+A^{1-x} X B^{x}\right\| d x \\
& \leq \frac{1}{8}\left\{\left\|A^{v} X B^{1-v}+A^{1-v} X B^{v}\right\|+2\left\|A^{\frac{3 v+1}{4}} X B^{\frac{3-3 v}{4}}+A^{\frac{3-3 v}{4}} X B^{\frac{3 v+1}{4}}\right\|\right.
\end{aligned}
$$




$$
\begin{aligned}
& +2\left\|A^{\frac{1+v}{2}} X B^{\frac{1-v}{2}}+A^{\frac{1-v}{2}} X B^{\frac{1+v}{2}}\right\|+2\left\|A^{\frac{v+3}{4}} X B^{\frac{1-v}{4}}+A^{\frac{1-v}{4}} X B^{\frac{v+3}{4}}\right\| \\
& +\|A X+X B\|\} .
\end{aligned}
$$

Remark 2.13 Putting $f(x)=\left\|\left|A^{x} X B^{1-x}\right|^{r}\right\| \cdot\left\|\left|A^{1-x} X B^{x}\right|^{r}\right\|$, where $\|\cdot\|$ is a unitarily invariant norm, $A, B, X \in B(\mathcal{H})$ with $A, B$ positive and $X \in \tau_{\|\cdot\| \cdot}$, and $r>0$. We first consider the case $0<v \leq \frac{1}{2}$. Since $f$ is decreasing on the interval $\left[0, \frac{1}{2}\right]$, we have

$$
f(v) \leq f\left(\frac{v}{2}\right)
$$

Using inequalities (12) and (16) for $f$ on the interval $[0, v]$, respectively, we get

$$
\begin{aligned}
& \frac{1}{2}\left\{f\left(\frac{v}{4}\right)+f\left(\frac{3 v}{4}\right)\right\} \\
& \quad \leq \frac{1}{4}\left\{f\left(\frac{v}{8}\right)+f\left(\frac{3 v}{8}\right)+f\left(\frac{5 v}{8}\right)+f\left(\frac{7 v}{8}\right)\right\}
\end{aligned}
$$

and

$$
\begin{aligned}
& \frac{1}{8}\left\{f(0)+2 f\left(\frac{v}{4}\right)+2 f\left(\frac{v}{2}\right)+2 f\left(\frac{3 v}{4}\right)+f(v)\right\} \\
& \quad \leq \frac{1}{4}\left\{f(0)+2 f\left(\frac{v}{2}\right)+f(v)\right\} .
\end{aligned}
$$

By the convexity of $f$ on the interval $[0, v]$ we have

$$
f\left(\frac{v}{2}\right) \leq \frac{f\left(\frac{v}{4}\right)+f\left(\frac{3 v}{4}\right)}{2}, \quad f\left(\frac{v}{2}\right) \leq \frac{f(0)+f(v)}{2} .
$$

Noting that

$$
\frac{f(0)+f(v)}{2}=\frac{f(1)+f(v)}{2} \leq f(1)=\left\||A X|^{r}\right\| \cdot\left\||X B|^{r}\right\|,
$$

by (i) of Theorem 2.11 we have

$$
\begin{aligned}
& \frac{1}{4}\left\{f\left(\frac{v}{8}\right)+f\left(\frac{3 v}{8}\right)+f\left(\frac{5 v}{8}\right)+f\left(\frac{7 v}{8}\right)\right\} \\
& \quad \leq \frac{1}{v} \int_{0}^{v} f(x) d x \\
& \quad \leq \frac{1}{8}\left\{f(0)+2 f\left(\frac{v}{4}\right)+2 f\left(\frac{v}{2}\right)+2 f\left(\frac{3 v}{4}\right)+f(v)\right\} .
\end{aligned}
$$

Combining inequalities (20), (21), (22), (23), (24), and (25), we have

$$
\begin{aligned}
f(v) & \leq f\left(\frac{v}{2}\right) \\
& \leq \frac{1}{2}\left\{f\left(\frac{v}{4}\right)+f\left(\frac{3 v}{4}\right)\right\}
\end{aligned}
$$




$$
\begin{aligned}
& \leq \frac{1}{4}\left\{f\left(\frac{v}{8}\right)+f\left(\frac{3 v}{8}\right)+f\left(\frac{5 v}{8}\right)+f\left(\frac{7 v}{8}\right)\right\} \\
& \leq \frac{1}{v} \int_{0}^{v} f(x) d x \\
& \leq \frac{1}{8}\left\{f(0)+2 f\left(\frac{v}{4}\right)+2 f\left(\frac{v}{2}\right)+2 f\left(\frac{3 v}{4}\right)+f(v)\right\} \\
& \leq \frac{1}{4}\left\{f(0)+2 f\left(\frac{v}{2}\right)+f(v)\right\} \\
& \leq \frac{f(0)+f(v)}{2} \\
& \leq f(0) .
\end{aligned}
$$

Similarly, when $\frac{1}{2}<v<1$, since $f(v)$ is increasing on the interval $\left[\frac{1}{2}, 1\right]$, we have $f(v) \leq$ $f\left(\frac{1+v}{2}\right)$. By the same process as before, we also have

$$
\begin{aligned}
f(v) & \leq f\left(\frac{1+v}{2}\right) \\
& \leq \frac{1}{2}\left\{f\left(\frac{3 v+1}{4}\right)+f\left(\frac{v+3}{4}\right)\right\} \\
& \leq \frac{1}{4}\left\{f\left(\frac{7 v+1}{8}\right)+f\left(\frac{5 v+3}{8}\right)+f\left(\frac{3 v+5}{8}\right)+f\left(\frac{v+7}{8}\right)\right\} \\
& \leq \frac{1}{1-v} \int_{v}^{1} f(x) d x \\
& \leq \frac{1}{8}\left\{f(v)+2 f\left(\frac{3 v+1}{4}\right)+2 f\left(\frac{1+v}{2}\right)+2 f\left(\frac{v+3}{4}\right)+f(1)\right\} \\
& \leq \frac{1}{4}\left\{f(v)+2 f\left(\frac{v+1}{2}\right)+f(1)\right\} \\
& \leq \frac{f(1)+f(v)}{2} \\
& \leq f(1) .
\end{aligned}
$$

Thus, inequalities (26) and (27) are refinements of the second inequality in (1) and also a refinement of Theorem 4 of Burqan [4].

Remark 2.14 Putting $g(x)=\left\|A^{x} X B^{1-x}+A^{1-x} X B^{x}\right\|$, where $\|\cdot\|$ is a unitarily invariant norm, and $A, B, X \in B(\mathcal{H})$ with $A, B$ positive and $X \in \tau_{\|\cdot\| \cdot}$. Replacing $f$ by $g$ in Remark 2.13, we obtain

$$
\begin{aligned}
g(v) & \leq g\left(\frac{v}{2}\right) \\
& \leq \frac{1}{2}\left\{g\left(\frac{v}{4}\right)+g\left(\frac{3 v}{4}\right)\right\} \\
& \leq \frac{1}{4}\left\{g\left(\frac{v}{8}\right)+g\left(\frac{3 v}{8}\right)+g\left(\frac{5 v}{8}\right)+g\left(\frac{7 v}{8}\right)\right\} \\
& \leq \frac{1}{v} \int_{0}^{v} g(x) d x
\end{aligned}
$$




$$
\begin{aligned}
& \leq \frac{1}{8}\left\{g(0)+2 g\left(\frac{v}{4}\right)+2 g\left(\frac{v}{2}\right)+2 g\left(\frac{3 v}{4}\right)+g(v)\right\} \\
& \leq \frac{1}{4}\left\{g(0)+2 g\left(\frac{v}{2}\right)+g(v)\right\} \\
& \leq \frac{g(0)+g(v)}{2} \\
& \leq g(0),
\end{aligned}
$$

where $0<v \leq \frac{1}{2}$.

Similarly, when $\frac{1}{2}<v<1$, we have

$$
\begin{aligned}
g(v) & \leq g\left(\frac{1+v}{2}\right) \\
& \leq \frac{1}{2}\left\{g\left(\frac{3 v+1}{4}\right)+g\left(\frac{v+3}{4}\right)\right\} \\
& \leq \frac{1}{4}\left\{g\left(\frac{7 v+1}{8}\right)+g\left(\frac{5 v+3}{8}\right)+g\left(\frac{3 v+5}{8}\right)+g\left(\frac{v+7}{8}\right)\right\} \\
& \leq \frac{1}{1-v} \int_{v}^{1} g(x) d x \\
& \leq \frac{1}{8}\left\{g(v)+2 g\left(\frac{3 v+1}{4}\right)+2 g\left(\frac{1+v}{2}\right)+2 g\left(\frac{v+3}{4}\right)+g(1)\right\} \\
& \leq \frac{1}{4}\left\{g(v)+2 g\left(\frac{v+1}{2}\right)+g(1)\right\} \\
& \leq \frac{g(1)+g(v)}{2} \\
& \leq g(1) .
\end{aligned}
$$

Therefore, inequalities (28) and (29) are refinements of the second inequality in (2) and also a refinement of Theorem 3 of Feng [5].

\section{Competing interests}

The authors declare that they have no competing interests.

\section{Authors' contributions}

All authors contributed equally to the writing of this paper. All authors read and approved the final version of this paper.

\section{Author details}

${ }^{1}$ College of Science, Shihezi University, Shihezi, 832003, P.R. China. ${ }^{2}$ College of Mathematics and Statistics, Chongqing University, Chongqing, 401331, P.R. China.

\section{Acknowledgements}

The authors would like to express their thanks to Editor and reviewers for their valuable comments and suggestions on our manuscript. This work is supported by the National Natural Science Foundations of China (Grant No. 11161040).

Received: 30 August 2016 Accepted: 21 October 2016 Published online: 04 November 2016

\section{References}

1. Hiai, F, Zhan, $X:$ Inequalities involving unitarily invariant norms and operator monotone functions. Linear Algebra Appl. 341, 151-169 (2002)

2. Bhatia, R, Davis, C: A Cauchy-Schwarz inequality for operators with application. Linear Algebra Appl. 223/224, 119-129 (1995)

3. Bhatia, R, Davis, C: More matrix forms of the arithmetic-geometric mean inequality. SIAM J. Matrix Anal. Appl. 14 132-136 (1993)

4. Burgan, A: Improved Cauchy-Schwarz norm inequality for operators. J. Math. Inequal. 10(1), 205-211 (2016)

5. Feng, Y: Refinements of the Heinz inequalities. J. Inequal. Appl. 2012, 18 (2012) 